\title{
Differential algebraic methods for single particle dynamics studies of the University of Maryland electron ring
}

\author{
E. Nissen* \\ Department of Physics, Northern Illinois University, DeKalb, Illinois 60115, USA \\ B. Erdelyi ${ }^{\dagger}$ \\ Department of Physics, Northern Illinois University, DeKalb, Illinois 60115, USA, \\ and Physics Division, Argonne National Laboratory, Argonne, Illinois 60439, USA \\ (Received 8 October 2009; published 12 July 2010)
}

\begin{abstract}
The University of Maryland electron ring is a small low energy machine for the study of space-charge dominated beams. Differential algebraic methods as implemented in COSY INFINITY offer an accurate method to study and analyze single particle nonlinear dynamics. As a starting point for space-charge related studies, we undertook a comprehensive examination of the single particle nonlinear dynamics based on differential algebra methods. Quantities such as tunes, chromaticities, dispersion, amplitude dependent tune shifts, and resonance strengths were calculated, and robustness of the solutions with respect to errors tested. The model demonstrated that the earth's magnetic field has a significant impact on the beam, and adds rich dynamics even in the absence of space charge. Initially we determined the tunes for which an injection-free idealization of the ring had the largest dynamic aperture. Our study then showed that the actual ring also had the largest dynamic aperture at these same tunes, and at these tunes was also least sensitive to errors. Comparison of predicted beam trajectories with measured data showed that the model was accurate for the examined area.
\end{abstract}

DOI: 10.1103/PhysRevSTAB.13.074001

PACS numbers: 41.75.Fr, 29.27.-a, 05.45.-a

\section{INTRODUCTION}

The University of Maryland electron ring (UMER) is an electron storage ring that is 3.8 meters in diameter, which uses low energy $(10 \mathrm{keV})$ electrons to study space-charge dominated beams, and that models some more costly heavy particle beam accelerators [1-3]. A necessary prerequisite for understanding space-charge dominated beams, and the efficient operation of the associated equipment, is the knowledge not only of the effects of space charge, but also the single particle nonlinear dynamics. During the design phase for the facility an analysis was performed [4], which measured the predicted effects of random errors in the placement and powering of the magnetic elements, as well as a preliminary look at how the beam might be steered in the earth's magnetic field. There has been steady progress with the operation of the ring [5-9]; however, there have been several changes to the design of the facility since its commissioning, as well as to the ambient magnetic fields surrounding it. So it is timely to perform a comprehensive nonlinear analysis of the current configuration. It is hoped that this will lead not only to a better understanding of the fundamental dynamics, but also increased operating efficiency.

The low energy of the beam and the small radius of the ring, combined with the earth's magnetic field, lead to

\footnotetext{
*enissen@gmail.com

†erdelyi@anl.gov
}

nonplanar reference trajectories and intricate relative motion. Furthermore, the injection method used involves deliberate misalignments which are difficult to model and optimize. Therefore examining the single particle dynamics of the as-built machine is a necessary endeavor.

The ring has 18 sections, see Fig. 1. Seventeen sections (labeled RC1 through RC17) comprise the main ring, each with two bending dipoles and two sets of focusingdefocusing quadrupoles, with a vertical steering dipole at the entrance. The last section (the $Y$ section) is where the beam is injected into the ring; this is accomplished by sending the beam through a large quadrupole and a pulsed dipole which changes bending direction based on whether the beam is being injected or is recirculating. There is a matching section that goes from the electron gun to the $Y$ section. This section contains a short solenoid with a unique field profile. Fourteen of these sections have diagnostic chambers in them which contain both destructive phosphor screens and nondestructive beam position monitors. Sections 4, 10, and 16 have glass gaps and section 10 contains the resistive wall current monitor used in this work [10]. For more details see [11].

The code used for this study was COSY INFINITY 9.0; for an overview of its capabilities see [12]. This code utilizes differential algebras (DA) to determine transfer maps to arbitrary order, and to analyze them by normal form methods [13]. It works by using DA vectors to calculate derivatives algebraically; this allows for exact computational differentiation. Since these methods allow for detailed 


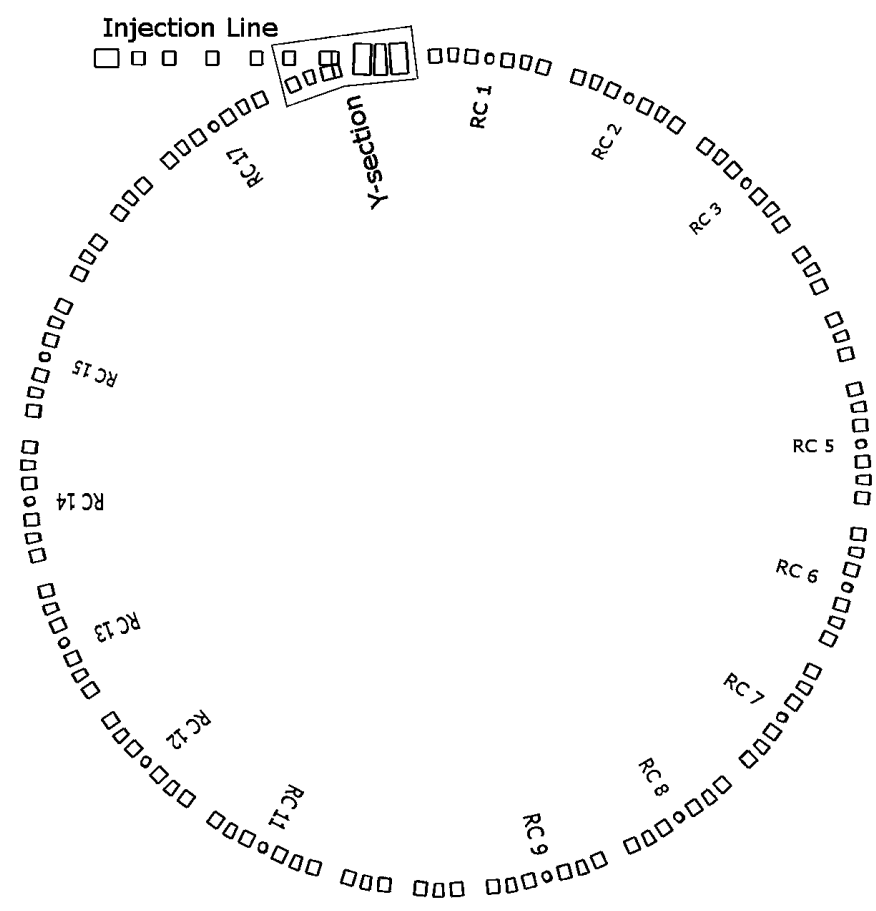

FIG. 1. A simplified diagram of the ring made using COSY INFINITY's graphics package. The sections labeled RC1, 2, etc. have diagnostic chambers containing phosphor screens and beam position monitors. RC4, 10, and 16 do not have beam position monitors to make room for other diagnostics.

maps to be calculated, quantities such as betatron tunes can be determined directly from the map without the need for lengthy tracking. In complicated systems the paths of particles as they move through phase space can trace out intricate patterns. Normal form methods perform a coordinate transformation such that the pattern left by the path of the particle after a large number of passes in a turn by turn surface of section is always a circle. Furthermore, since the DA methods can be used to find the derivatives with respect to any variable we wish, the chromaticities can be directly calculated by making energy a variable. Since the normal form method is a coordinate transform, individual particles can have their tunes directly determined, allowing for the computation of amplitude dependent tune shifts. This also allows for a study of the resonances, and the resonance strengths can be directly computed [13]. COSY also has an architecture which allows new elements to be easily implemented. This can be used either for elements with unusual properties such as the UMER short solenoid, or for adding effects to existing elements such as kicks from the earth's magnetic field or image charges. Furthermore, COSY has the ability to treat arbitrary misalignments exactly.

The main types of studies performed using COSY involved steering of the beam, tune measurements, resonance strength calculation, and amplitude dependent tune shift calculation. In addition, a range of operating points which give different horizontal and vertical tunes were calculated and compared for effectiveness in maintaining the beam quality.

The outline of the paper is as follows: Section II contains the theoretical background and various computer studies of the ring that were performed using COSY INFINITY. Derivations of the effects of the earth's magnetic field and image charges are shown in Appendices A and B. Section III shows the results of a number of experiments done in the University of Maryland electron ring, and a comparison to their predicted values. Finally, in Sec. IV we conclude with a brief summary.

\section{MODELING AND SIMULATIONS}

\section{A. Theoretical background}

The UMER beam was modeled in COSY in a manner that involved effects from the earth's magnetic field as well as the effects of image charges. The vertical component of the earth's field is the strongest, and acts to provide roughly $20 \%$ of the bending in the ring. Hence, this effect, negligible in almost all accelerator applications, becomes significant for UMER. COSY's default routines produce transfer maps for standard machine elements, all of which entail planar reference orbits. This is not the case when the earth's field and image charge effects are included. This involves altering some of COSY's procedures. These effects were added by introducing kicks at regular intervals within each element. These kicks were enacted using Strang splitting $[14,15]$. This method works for any set of differential equations, and does not necessarily require them to be Hamiltonian. Since the dominant forces in this effect are the horizontal and vertical magnetic fields, an $A_{z}$ can always be derived to account for these fields, and symplecticity is not an issue. While there is a small longitudinal field COSY has a routine that will symplectify the map [16].

Strang splitting is a way of taking a system where there are multiple physical effects that can each be separately described by a differential equation in the coordinates $\vec{z}=$ $(\vec{q}, \vec{p})$ :

$$
\begin{aligned}
& \frac{d \vec{z}}{d s}=\vec{g}_{1}(\vec{z}, s) \Rightarrow \vec{f}_{1}(s), \\
& \frac{d \vec{z}}{d s}=\vec{g}_{2}(\vec{z}, s) \Rightarrow \vec{f}_{2}(s),
\end{aligned}
$$

where $s$ is the independent variable and $\vec{g}_{1}(\vec{z}, s)$ and $\vec{g}_{2}(\vec{z}, s)$ are arbitrary smooth vector functions, and the initial conditions are known. While the method can be adapted for nonautonomous systems [17-19], for numerical purposes the system was broken into sections small enough to be considered autonomous. Assuming known solutions $\left[\vec{f}_{1}(s), \vec{f}_{2}(s)\right]$ to the separate equations, Strang splitting shows that a good approximation of the solution is 


$$
\frac{d \vec{z}}{d s}=\vec{g}_{1}(\vec{z})+\vec{g}_{2}(\vec{z}) \Rightarrow \vec{f}_{2}\left(\frac{s}{2}\right) \circ \vec{f}_{2}(s) \circ \vec{f}_{1}\left(\frac{s}{2}\right)+\mathcal{O}\left(s^{3}\right),
$$

which gets increasingly accurate with decreasing $s$.

In order to change as little as possible the existing COSY elements, we apply Strang splitting with the following identifications:

$$
\begin{aligned}
\vec{f}_{1}(s) & \mapsto \mathcal{M}(s), \\
\vec{f}_{2}(s) & \mapsto \mathcal{K}(s),
\end{aligned}
$$

where $\mathcal{K}$ is a kick (due to the earth's field or image charge) and $\mathcal{M}$ is the COSY generated map for the element, and $s$ is (a fraction of) the length of the element. The strengths for the kicks are derived from magnetic field data, and the geometry of the ring in Appendices A and B.

COSY INFINITY can calculate a number of quantities using normal form methods. Normal forms are just a coordinate transformation from the particle optical coordinates $\vec{z}=(\vec{q}, \vec{p})$ to action angle coordinates $\vec{j}$. This is done with a transformation $\mathcal{A}$, such that $\vec{j}=\mathcal{A}(\vec{z})$. When these action angle coordinates undergo a transformation $\mathcal{N}$ they move in circles with radius $|\vec{j}|$. The normal form $\mathcal{N}$ is related to the original map $\mathcal{M}$ by the relation $\mathcal{N}=$ $\mathcal{A} \circ \mathcal{M} \circ \mathcal{A}^{-1}$. This transformation generates a map of the form

$$
\mathcal{N}=\left(\begin{array}{ccc}
\mathcal{N}_{1} & & 0 \\
& \mathcal{N}_{2} & \\
0 & & \mathcal{N}_{3}
\end{array}\right)
$$

where

$$
\mathcal{N}_{m}=\left(\begin{array}{cc}
\cos \left[\theta_{m}(\vec{j})\right] & \sin \left[\theta_{m}(\vec{j})\right] \\
-\sin \left[\theta_{m}(\vec{j})\right] & \cos \left[\theta_{m}(\vec{j})\right]
\end{array}\right) .
$$

This makes $\theta$ of the form $\theta(\vec{j})=\theta_{0}+a j_{1}+b j_{2}+$ $c j_{1} j_{2}+d j_{1}^{2}+\cdots$, where $\theta_{0}$ is the tune and $a, b, c, d, \ldots$ are the amplitude dependent tune shifts. If energy is declared a parameter $\delta$, i.e. $\theta_{0} \rightarrow \theta_{0}(\delta)$, then there will be an expansion $\theta_{1} \delta+\theta_{2} \delta^{2}+\cdots$, where $\theta_{1}$ is the chromaticity. The normalizing map $\mathcal{A}$ gives the resonance driving terms, and can be used to obtain the matching conditions [13].

\section{B. Simulation studies}

Since high fidelity simulations are desired, the default COSY elements were used as much as possible. However, changes were required to simulate novel elements and effects. The quadrupoles were implemented using a hard edge model that was developed previously $[20,21]$. While a detailed examination of the fringe fields was performed before the machine was built $[22,23]$, this particular study restricts itself to the integrated hard edged model, since this lends itself well to the addition of the earth's magnetic field

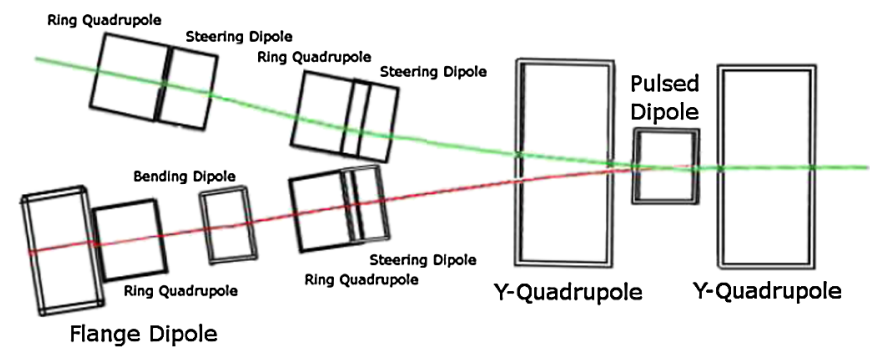

FIG. 2. (Color) A diagram of one particular operating point steering the beam through both injection (green) and recirculation (red) sections.

and measured fringe field data was unavailable. One of the complicating issues in UMER is its method of injection and recirculation. The design sends the beam through the first quadrupole deliberately off-center, so it acts both as a focusing element and as a bending element; see Fig. 2. COSY's ability to directly implement offsets to the beam line was beneficial in modeling this behavior, but since the beam is deliberately off-center, the matching and steering of the beam become interlinked processes.

Another difficulty stems from assumptions COSY makes regarding the nature of the reference orbit. COSY assumes that all reference trajectories are planar, while the earth's field can alter the trajectory into all three dimensions. Because of this nonplanar nature, adding the earth's field to the simulation can be done with kicks; see Sec. II A. The magnetic field and image charge kicks are applied in the different elements at different rates; the quadrupoles have 15 kicks per element, while dipoles and drifts have six kicks per element. The number of kicks was determined by increasing the number of kicks until the output converged to the precision of the measured earth's field (approximately $10^{-4}$ Gauss). The image charge kicks are included within the $Y$ section due to the significant offset in that region. The same number of kicks per element was enough for convergence. The values for the components of the earth's field at the location of the kicks were determined by interpolating values measured at each dipole. The paths of the injected and recirculated reference orbits around the $Y$ section are shown in Fig. 2.

The beam was modeled with a uniform spatial distribution and a Maxwellian velocity distribution, starting at the cathode with the spatial domain determined by the size of the cathode, and the velocity distribution determined by the temperature. After the beam was modeled in COSY INFINITY, a series of simulations were run. The simulations were performed in such a way that they took more and more effects into account from the various ordered terms. First we studied the 0th order effects, steering through the matching section and ring. Then 1st order effects were taken into account: the injection section was matched, betatron tunes were calculated, as was the chromaticity, the momentum compaction, and the dispersion. Finally, nonlinear effects were taken into account, including am- 
plitude dependent tune shifts, higher order chromaticities, and resonance strengths. However, it should be noted that while we talk about 0th and 1st order effects, the higher order effects on the motion of the beam will effect the final values of the computed terms, since the reference orbit is off-centered.

\section{Constant term effects}

In order for the beam to be used for long term studies, accurate steering both at injection and during recirculation is necessary. Steering through the injection line is accomplished using steering dipoles, while steering throughout the ring is accomplished by varying the current through the bending dipoles. Determining a closed orbit that stays close to the centerline of the machine is important, and the earth's magnetic field makes this task much more difficult. The initial round of simulations involved steering through each two-dipole section by assuming that the beam would enter the section at the center of the beam pipe with zero lateral velocity, and the dipoles were changed such that the beam would exit the section at the center of the beam pipe also with zero lateral velocity. Also, the beam would enter and leave each section moving straight through the section without any angle with respect to the centerline of the beam pipe. The results shown in Fig. 2. are the product of extensive optimizations using COSY's built in optimization algorithms.

Previous steering solutions [24] for the ring had used the earth's magnetic field data to try to reduce the dipole currents by an amount equal to the equivalent of the integrated earth's field, which gave similar predictions as can be seen in Fig. 3. From an operations standpoint, the relative smoothness or jaggedness of the current settings are inconsequential. Attempts that were made to smooth (global, polynomial, running averages, etc.) deteriorated the steering of the beam. The ring steering as calculated with COSY assumed that the beam centroid would enter the first turn at the center of the pipe with zero angle, and a fitting algorithm was used to have it exit the turn at the center of the pipe with zero angle. Therefore the steering algorithm was designed such that the beam centroid would leave the electron gun aperture and enter the ring under those conditions. This involves both the steering dipoles and the matching quadrupoles working together to bring the beam into the ring. For recirculation the simulations used a combination of the bending dipole on the recirculation side of the ring, the recirculation steering dipole, and the pulsed dipole. All elements are at fixed locations. The purpose of this optimization was to make all perturbations as local as possible. An example of the closed orbit for one particular operating point is shown in Fig. 4. In this figure it can be seen that with a good set of dipole corrections the deviation of the closed orbit from the center can be kept in the horizontal plane to the submillimeter scale. The number of vertical steering degrees of freedom made optimization of the vertical trajectory more challenging. One method that increased the speed at which a solution was found, was to add the position and angle terms to the objective function in quadrature. In the vertical direction the displacement is a few millimeters.

\section{Linear effects}

Next, the linear behavior of UMER was examined, to try to determine the best operating point. The matching settings for the injection line were determined using a set of 5000 particles arranged for a given aperture that were sent

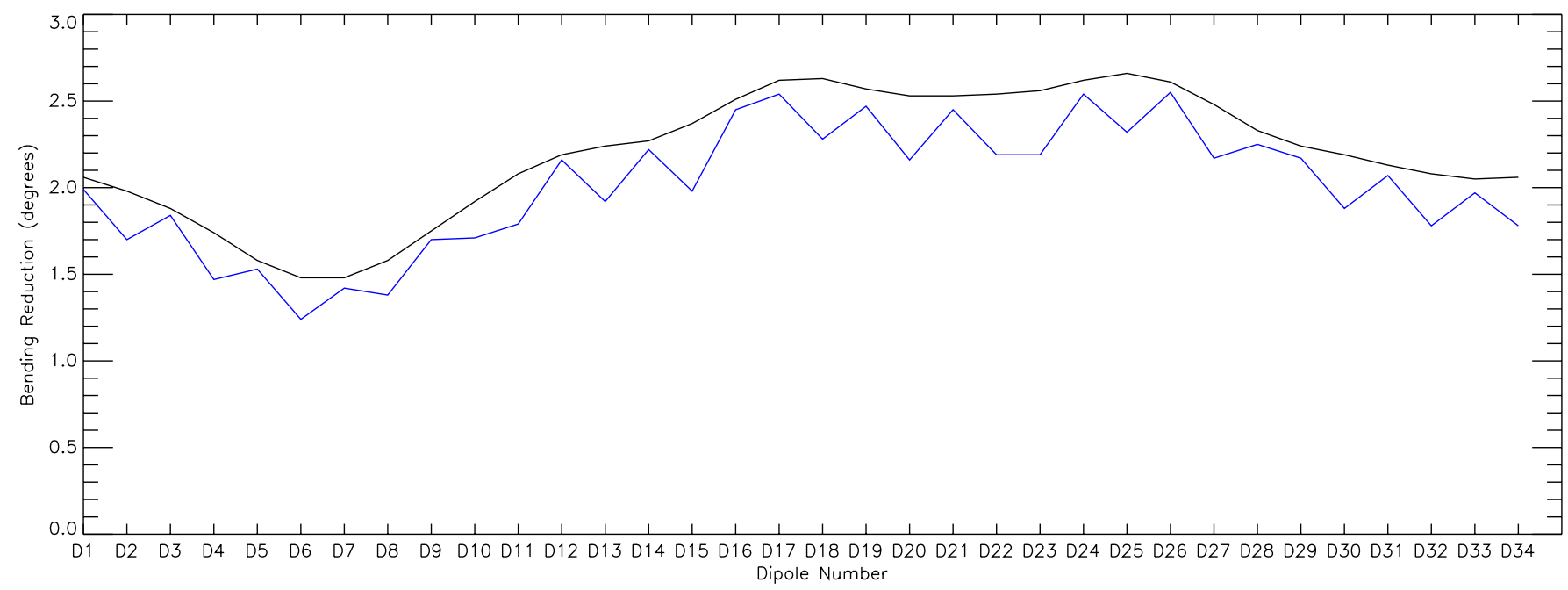

FIG. 3. (Color) A comparison of values for the ring steering. In order to account for the earth's magnetic field, the ring bending dipoles must be altered from their physical bending setting of 10 degrees. This is a plot of that change. The black line is the previous method of reducing the dipole currents by the amount equal to the equivalent of the integrated magnetic field over the section. The blue line is the result of having COSY INFINITY steer the beam to the center of the pipe with zero angle at the end of each focusing-defocusing (FODO) section. We attempted to smooth out the settings but every attempt made the steering significantly worse. 

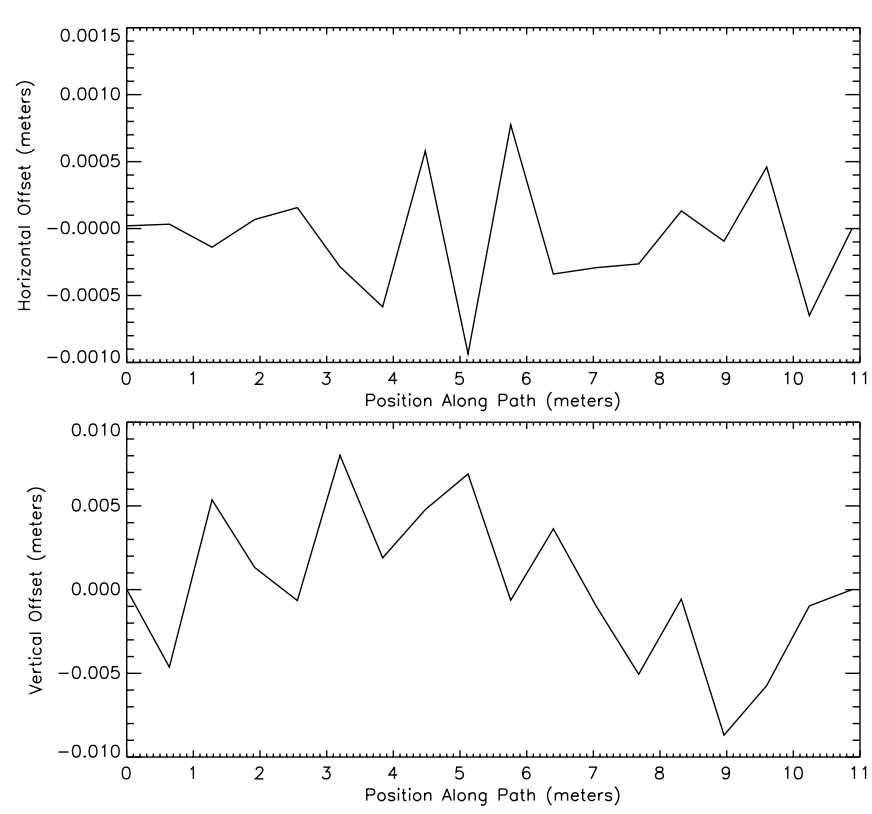

FIG. 4. A plot of the closed orbits for one operating point both horizontally and vertically.

through the solenoid, then matched to the ring's Twiss parameters as generated by COSY, and the steering dipoles were then fitted to move the beam through the injection line to the center of the beam pipe with zero angle. The two quadrupoles in the injection section keep the same values that were determined for the ring matching, but the pulsed dipole can be varied to steer the beam towards its closed orbit. The matching algorithm uses the linear elements of the map, and takes linear coupling into account. This was performed with COSY's internal optimization algorithms.

The next set of simulations used the large quadrupoles in the $Y$ section to control the betatron tune. The tune that would result if instead of an injection section with 17 ring sections the ring was made of 18 identical sections (i.e. the optimal value for the fully symmetric layout) is called the ideal tune. We use the magnetic field, quadrupole and dipole settings of the other 17 sections combined with a similar set for the 18th section. The ideal tunes were calculated to be $\left(\mu_{1}, \mu_{2}\right)=(6.76457,6.63717)$. The differences in the quadrupole strengths greatly affect the steering of the beam since the beam enters them off-center. Therefore the tune matters not only as the operating point, but also as part of the steering. We see that the $x$ tune can be varied more according to this study than the $y$ tune. A total of 81 values for the betatron tune, centered around the ideal tune, were investigated to determine how much beam remained within the beam pipe, a measure of the dynamic aperture, after a set number of turns. These values cover a square of tunespace with a magnitude of 0.08 per side. This method of measuring the dynamic aperture involved creating a set of initial conditions at the location of the electron gun's aperture, and following the particles through the beam line as they go through injection and into the ring

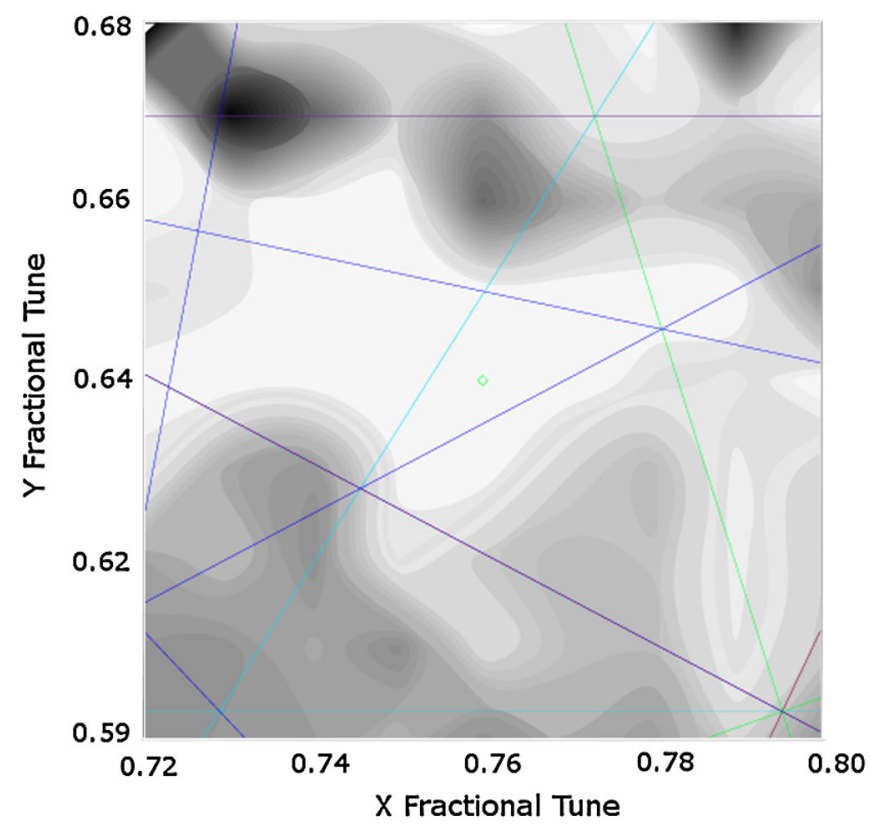

FIG. 5. (Color) A contour plot of the scanned tune space. Darker areas denote less of the beam surviving after 100 turns. The green $\diamond$ symbol represents the location of the ideal tunes. Superimposed are the resonance lines up to sixth order, red indicates 2nd order, orange indicates third order, green indicates fourth order, light blue indicates fifth order, and dark blue indicates sixth order.

for a set number of turns, the number of initial conditions that remain in the beam pipe $(2.95 \mathrm{~cm}$ radius $)$ are used to determine the dynamic aperture. The initial conditions had a uniform distribution in space, using the cathode radius of $4 \mathrm{~mm}$ and were Gaussian in angle distribution, using a temperature of $1100^{\circ} \mathrm{C}$. In the study shown in Fig. 5 that number could be as much as $100 \%$ of the particles, or as low as $0 \%$. Large differences are seen in the survival rates of the different tunes. The best operating point seems to be in the vicinity of the ideal tune. Figure 5 also has the low order resonance lines shown in it; it should be noted that this contour plot is created by interpolating a grid of 81 operating points. This means that, even if a low order

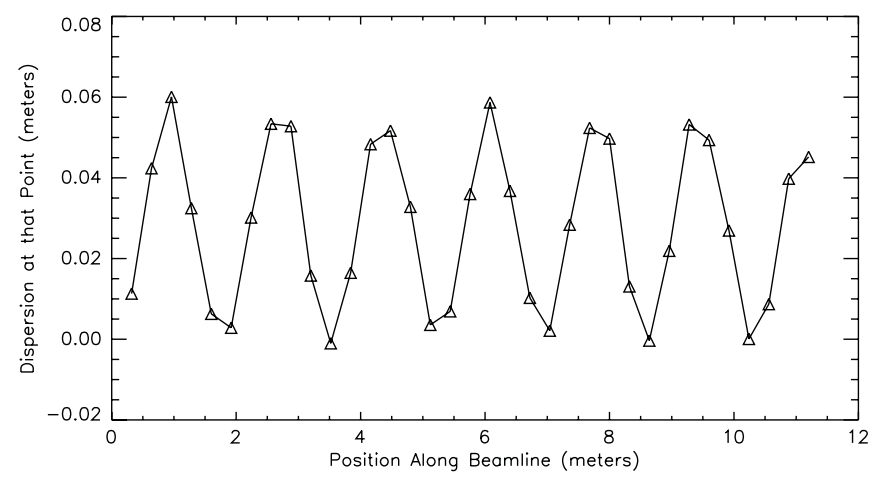

FIG. 6. A plot of the progression of the dispersion as the one term map is generated FODO section by FODO section. 


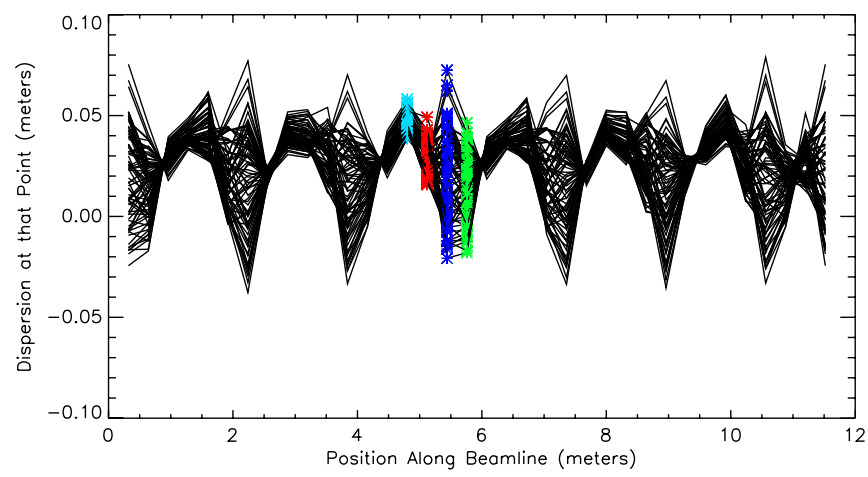

FIG. 7. (Color) A plot showing the dispersion of the 81 operating points used in this study, all are superimposed to show the variability in the dispersion of the one-turn map as calculated around different points of the ring. The colors denote the positions of the contour plots shown in Fig. 8 in order.
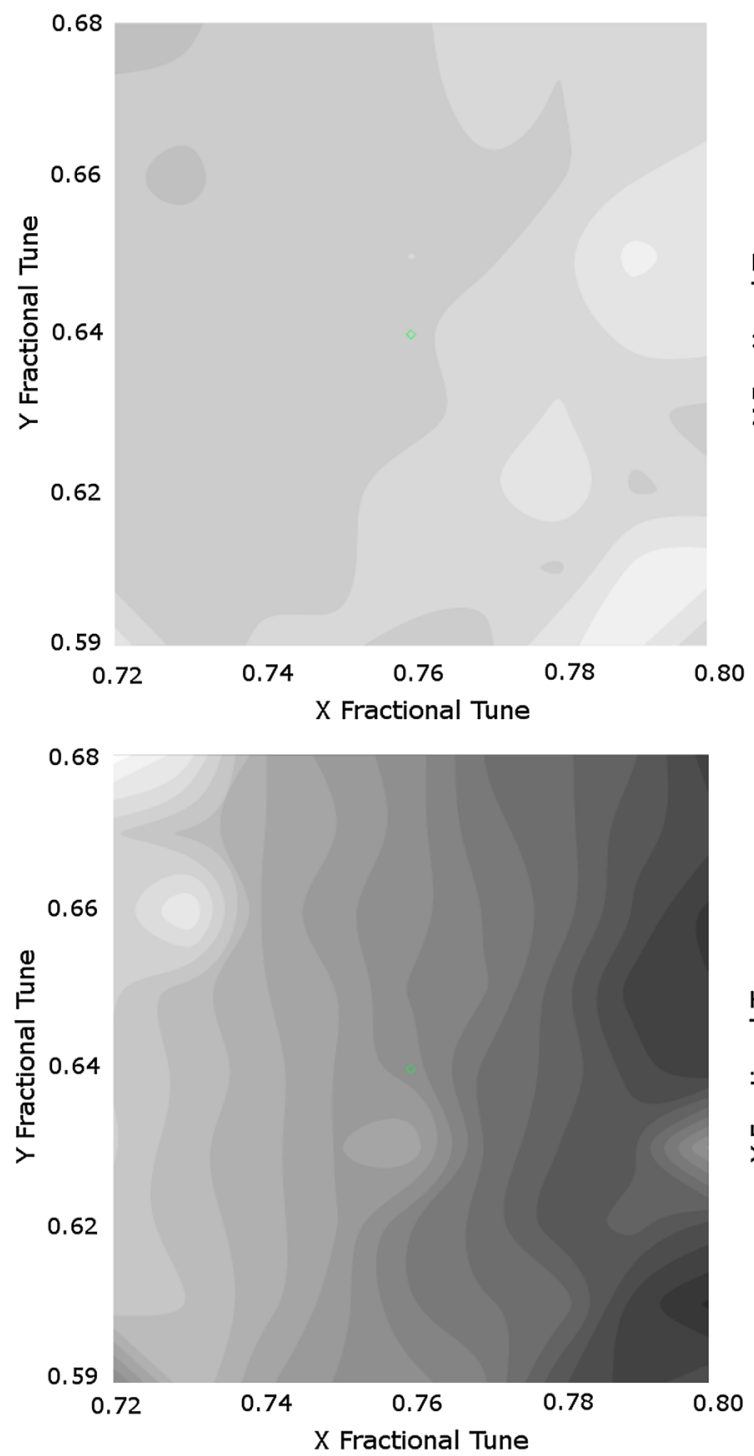

resonance seems to show a small effect, that does not mean that it is weak, only that there was no operating point calculated on that resonance.

Another quantity that needs to be understood is the maximum dispersion of the various operating points. Since the dipole settings within the ring remain the same for all of the operating points, it is the steering settings through the $Y$ section that contribute to the differences. It is also informative to investigate the manner in which the dispersion changes as the map is generated element by element; this behavior is shown in Fig. 6. Furthermore, if the beam is measured at various places around the beam the levels of dispersion will differ; the behavior of the oneturn dispersion element is plotted as a function of distance around the ring in Fig. 7. There is a distinct repetitive pattern to the dispersions of the various maps as we
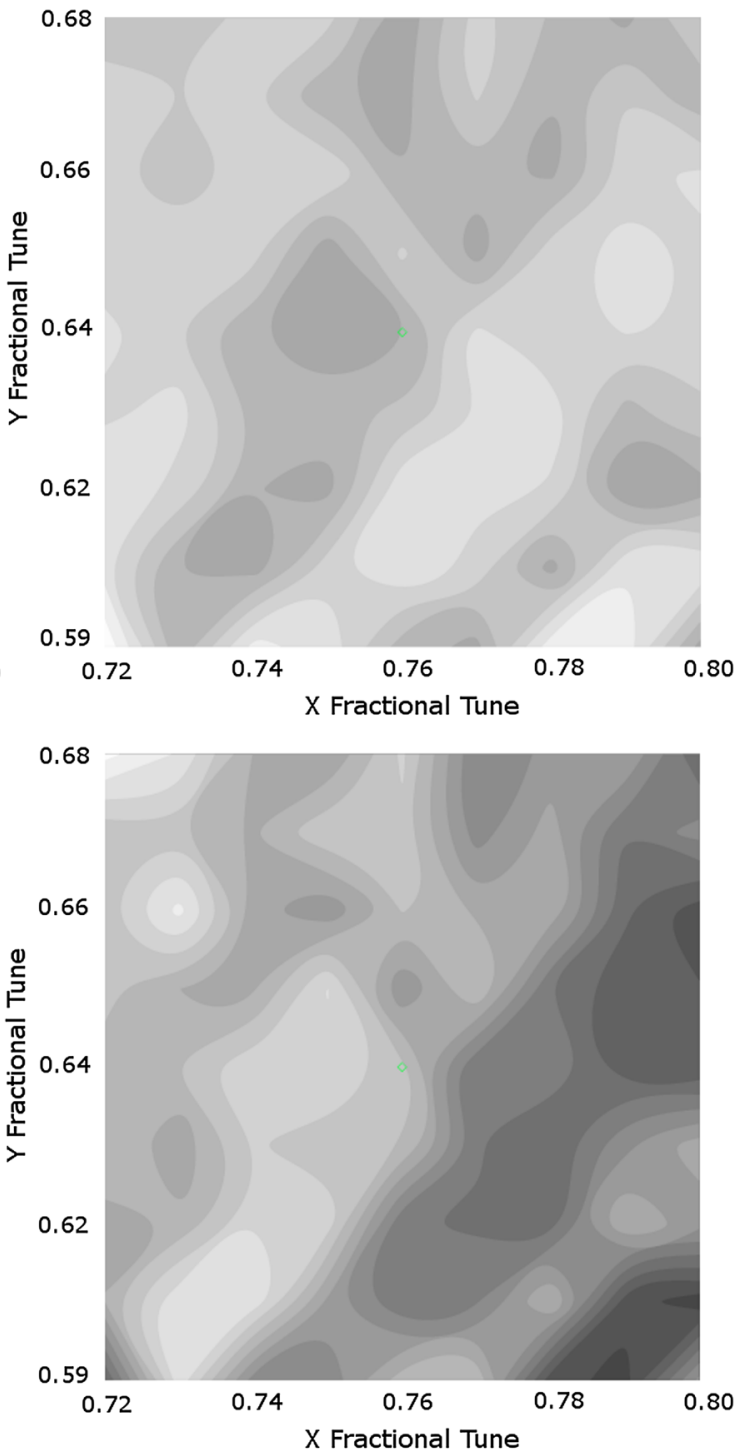

FIG. 8. Four contour plots of the dispersion across the scanned tune space. Darker areas denote lower levels of dispersion. The values are contoured between $-0.02 \mathrm{~m}$ and $0.08 \mathrm{~m}$. The locations for the upper left, upper right, lower left, and lower right are marked with the light blue, red, dark blue, and green points in Fig. 7, respectively. The green $\diamond$ symbol represents the location of the ideal tunes. 


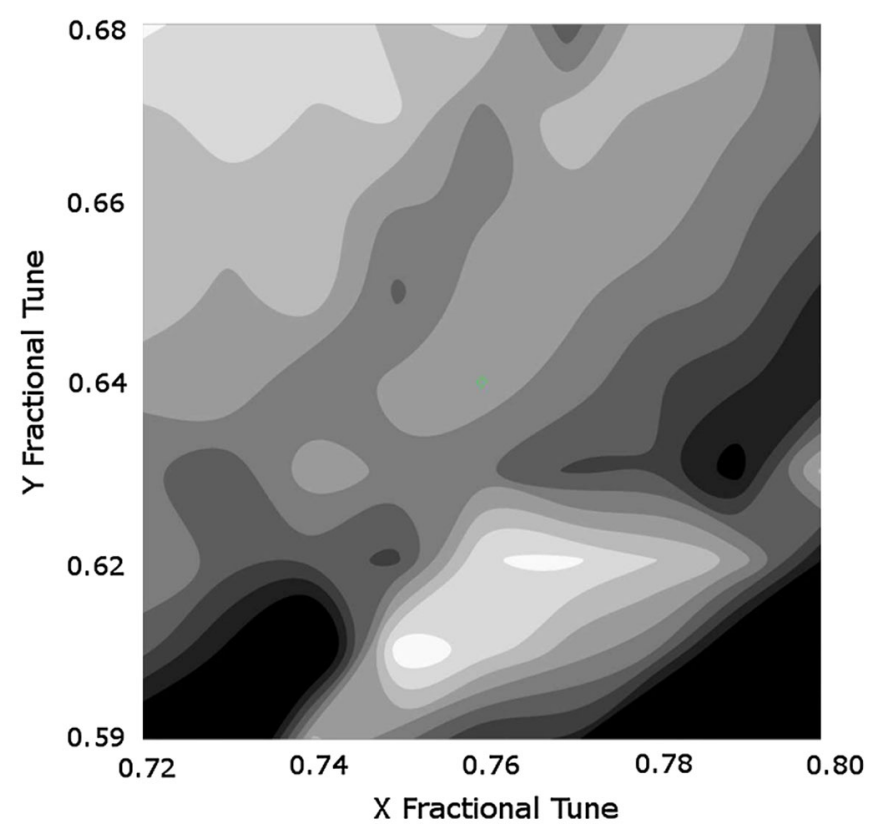

FIG. 9. A contour plot of the momentum compaction across the scanned tune space. Darker areas denote a smaller compaction factor. The range of values is between -0.0006 to 0.0005 . The green $\diamond$ symbol represents the location of the ideal tunes.

move around the ring, with the range of values going through periods being small, and then quite large. A comparison of the manner in which this range of values is distributed with respect to tune space is shown in Fig. 8. These four plots show that the morphology of the distribution in tune space is not a function of the range of variables, since the two on the left side share similar morphologies, while have very different ranges of values.

It is also possible to measure the momentum compaction of the operating points; the comparison is shown in Fig. 9. The momentum compaction values range from -0.0006 to 0.0005 and can be seen to be connected to both the $x$ and $y$ tunes.

\section{Nonlinear effects}

Using the DA normal form methods that are an integral part of COSY, it is very simple to calculate the chromaticities. The chromaticities are shown in Table I. These compare the calculated values for four operating points that were the default settings used for four currents during March of 2009. The labels are historical in nature, as the beam current in the simulation only affects the image charge force, which has a very small effect. The reason these settings are different is that they must be matched in a different manner which affects the steering through injection and ultimately the betatron tune. The addition of the higher order terms can help further distinguish operating points; if we compare the chromaticities for the pencil beam and the $7 \mathrm{~mA}$ beam, the pencil is only larger by $57 \%$ whereas the next term is not only 4 times larger in
TABLE I. Betatron tune predictions for four operating points. The first column contains the order of the term: 0th order is the tune itself, 1 st is $\frac{d \mu}{d \delta}, 2$ nd is $\left(d^{2} \mu\right) /\left(d \delta^{2}\right)$ etc. The second column is the value predicted by COSY. The four operating points are labeled above the terms.

\begin{tabular}{lccc}
\hline \hline Pencil beam & \multicolumn{3}{c}{$7 \mathrm{~mA}$ beam } \\
Order & $\mu(\delta)$ & Order & $\mu(\delta)$ \\
0 & 0.747 & 0 & 0.63722 \\
1 & -7.1058 & 1 & -4.4947 \\
2 & 111.479 & 2 & -25.83 \\
3 & -4787.3359 & 3 & -969.6789 \\
\hline $23 \mathrm{~mA}$ beam & \multicolumn{4}{c}{$80 \mathrm{~mA}$ beam } \\
Order & $\mu(\delta)$ & Order & $\mu(\delta)$ \\
0 & 0.6796 & 0 & 0.6900 \\
1 & -6.172 & 1 & -5.0174 \\
2 & -11.2667 & 2 & -31.2928 \\
3 & 2034.3401 & 3 & -1221.4241 \\
\hline \hline
\end{tabular}

magnitude, but also opposite in sign. The beams were distinguished by peak current, with the pencil beam being the lowest at $0.6 \mathrm{~mA}$. In the simulations the chromaticities depend heavily on the quadrupoles in the $Y$-injection section, as that was the major change for the repetitive portions of the ring.

The strengths of the various resonances can also be calculated. In Fig. 10 the resonance strengths for four operating points are shown; the $7 \mathrm{~mA}$ operating point has smaller resonance strengths than the others. The four operating points in Fig. 10 have different steering and magnet settings which lead to different resonances being dominant. However, the $(1,2),(1,0)$, and $(2,1)$ resonances are prominent in each operating point, which is most likely the resonances excited by the 17 ring sections since these are not changed as significantly as the injection line and the $Y$ section between the operating points. Note the absence of large high order resonances in any of the operating points. It is interesting to note that operationally the $7 \mathrm{~mA}$ beam seems to be the most well-behaved beam.

Using the normal form methods available in COSY, it is possible to calculate amplitude dependent tune shifts; these lead to footprints of the kind seen in Fig. 11. Other operating points have different patterns, and some do not display this behavior altogether. Within the tune scan that was performed as part of this analysis, it was the operating points with higher $y$ tunes that showed the fewest examples of amplitude dependent tune shifts, and when they were calculated for default UMER settings the ones detected were very small.

Another issue that would be of concern when selecting an operating point is the effect of errors on the beam. This study used the 81 operating points that had been determined previously. Both the effects of placement errors of the elements and of the strengths of the magnets were simulated. These effects were calculated using the design 

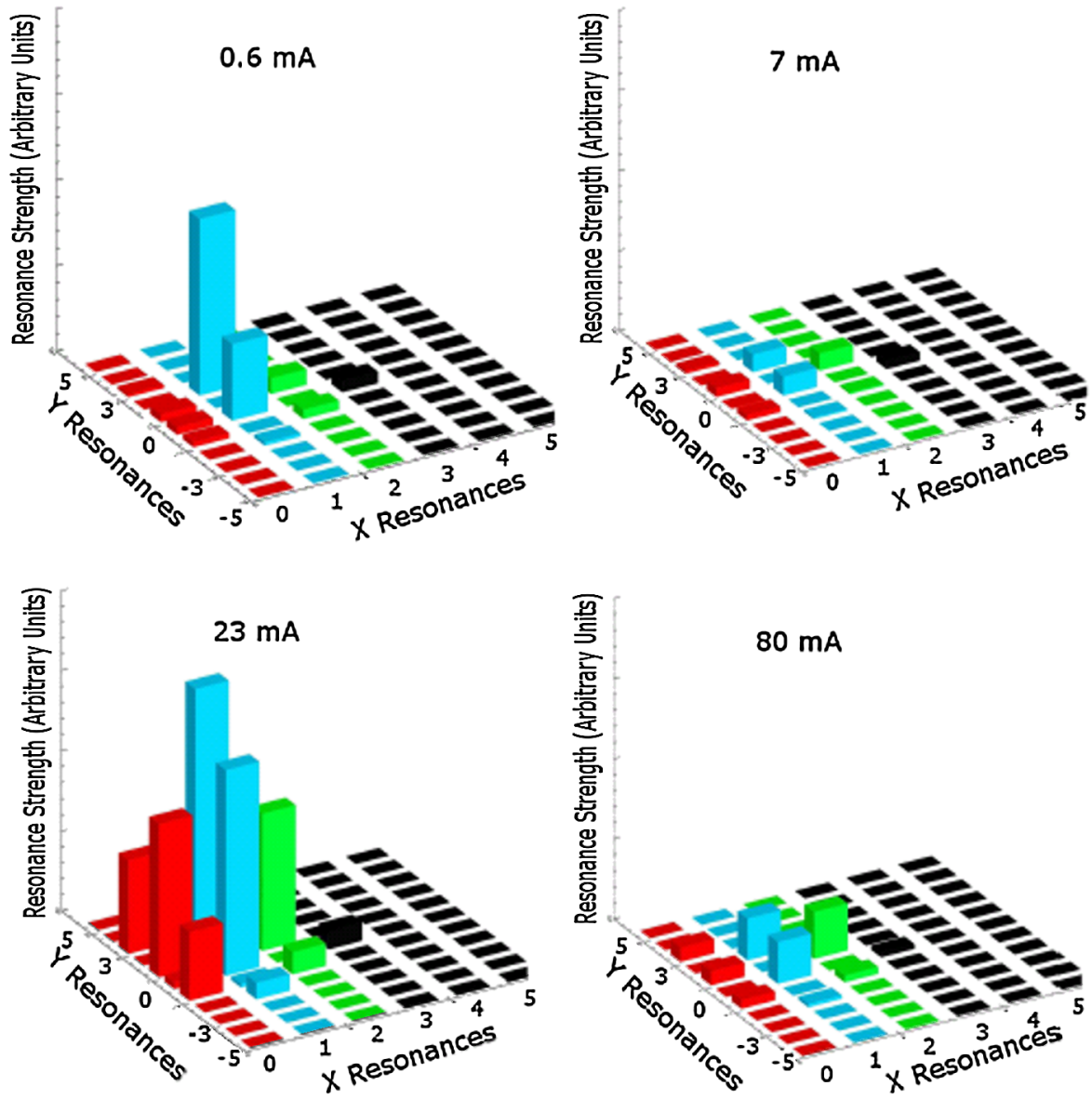

FIG. 10. (Color) A plot of up to sixth order resonance strengths for the four current values examined. Strengths are based on a $50 \mu \mathrm{m}$ emittance and all vertical axes are to the same scale. Colors are for visual differentiation only.
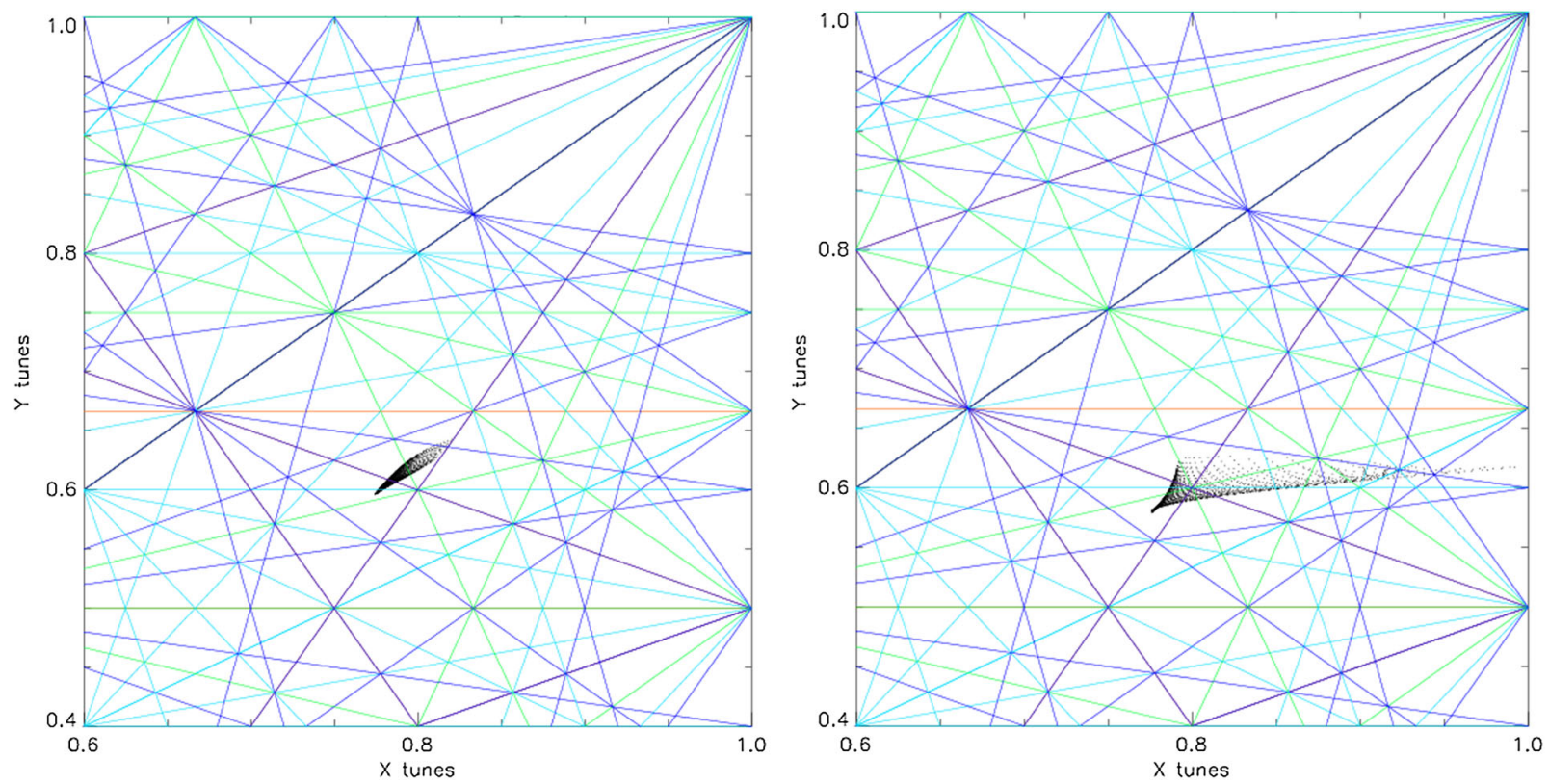

FIG. 11. (Color) The left side shows a well-behaved tune footprint, while the right side shows a more diffuse tune footprint. The differences are caused by different quadrupole settings. The lines are the resonance lines up to order six, with the same color scheme as Fig. 5. 
tolerances for the ring, by creating 100 sets of combined offsets in the ring, in essence there were 100 slightly different rings. These offsets were based on the design tolerances as set for the beam during the design phase [4]. The placement offsets for horizontal and vertical had a standard deviation of $0.1 \mathrm{~mm}$, the displacement along the centerline had a standard deviation of $1.0 \mathrm{~mm}$, and the rotation of the elements around the axis of the beam pipe had a standard deviation of $4.0 \mathrm{mrad}$. The static magnet current errors had a standard deviation of $0.5 \%$. The same set of initial conditions was sent through each of these rings, and the standard deviation for the $x$ direction was calculated for each set of initial conditions. After averaging for each operating point, the results for the placement offsets are shown in Fig. 12 and for the magnetic field in Fig. 13. As is readily visible, the areas that are least susceptible to magnet current errors are also least susceptible to placement errors; these indicate operating points which are more robust within the range of errors anticipated when the machine was built.

The hard edged model used here includes integrated effects of the fringe field. An attempt was made to use the default fringe field routines that come with COSY; however, this led to a number of issues since the routines are designed for much larger elements that are spaced further apart. In the UMER case there is overlapping of the fringe fields of different elements. While a theoretical treatment was made before UMER was built [22,23], measured fringe field data was unavailable. Since experi-

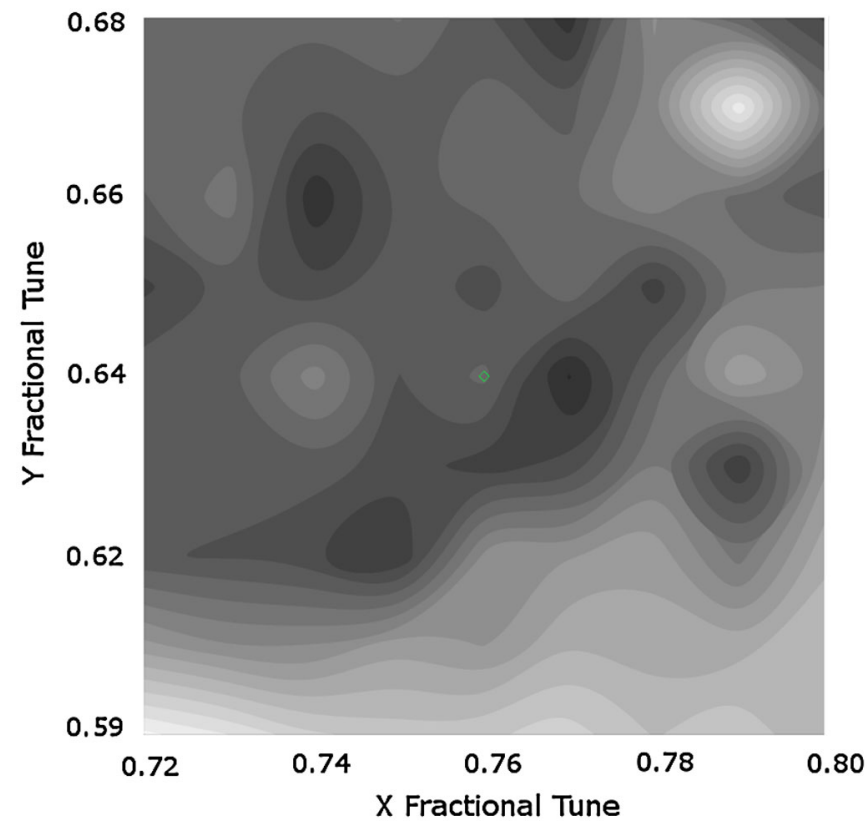

FIG. 12. A contour plot of the average standard deviation for 100 different sets of placement offsets. The darker areas show a smaller standard deviation of offset, which means they are less sensitive to errors. The green $\diamond$ symbol represents the location of the ideal tunes.

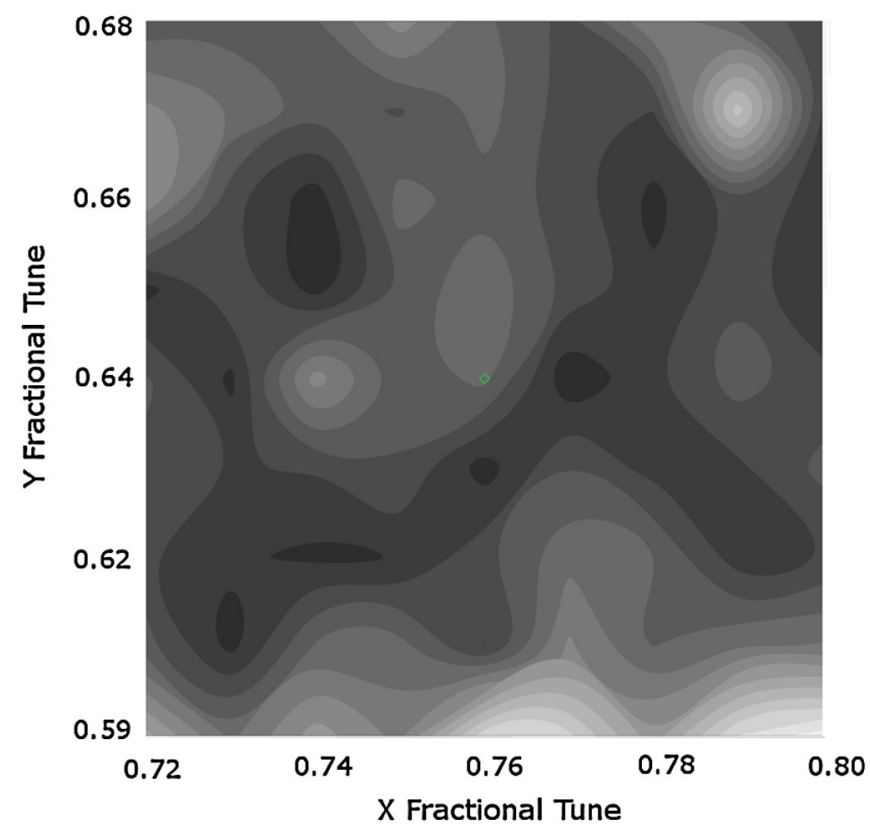

FIG. 13. A contour plot of the average standard deviation for 100 different sets of magnet current offsets. The darker areas show a smaller standard deviation of offset, which means they are less sensitive to errors. The green $\diamond$ symbol represents the location of the ideal tunes.

mental data was unavailable and interesting nonlinear dynamics were found without fringe fields, the analysis here involves only the hard edged approximation.

This initial set of simulations shows the great importance of including the earth's field in these calculations. The earth's field continuously deflects the beam out of its centered orbit requiring intricate steering solutions, which can only imperfectly keep the beam centered. This will then affect the way that the beam is focused since it is no longer necessarily entering the quadrupoles symmetrically. In effect the earth's field makes UMER a drift-free storage ring. An examination of the dynamic aperture for a model of UMER that does not use the earth's field shows $100 \%$ transmission of the beam across all 81 operating points, as compared to Fig. 5.

Figures 5, 12, and 13 imply that the region around the ideal tune will be the best to use: it has the largest dynamic aperture and the highest resilience with respect to errors. Finally, these simulations show that, even if the ideal tune cannot be obtained, in a small region of tune space surrounding the ideal tune, the machine will still work with minimal beam loss, even with the complicating factors of the earth's magnetic field, image charges, and design offsets.

\section{EXPERIMENT AND COMPARISON}

We performed a series of experiments on the ring in an effort to benchmark the simulations. The first experiment was to compare the results of the steering solution provided 


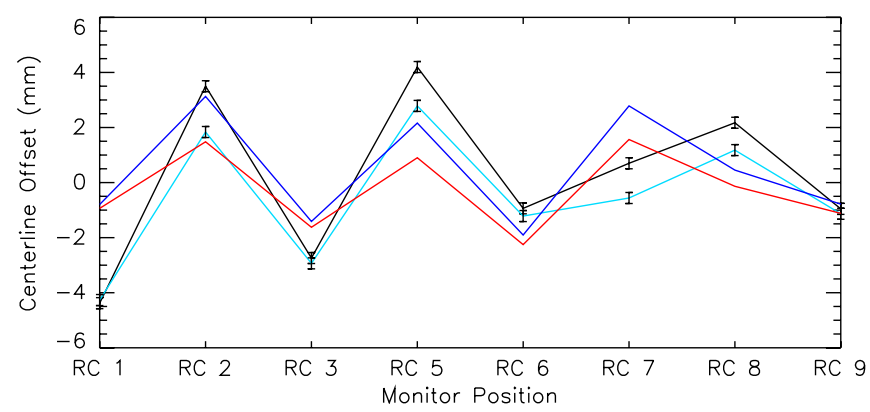

FIG. 14. (Color) A comparison of the predicted and measured ring steering trajectories. The black line is the previous magnetic field compensation value, while the light blue line is the one calculated in this study. The dark blue line is the predicted trajectory for the previous compensation value, while the red line is the predicted trajectory for the settings determined in this study.

by COSY and the previous magnetic field compensation values, as described in Sec. II B. The results of simulating both steering settings as well as their measured values are shown in Fig. 14. We see fairly good agreement between the predicted and measured values as they progress around the ring. The relative movement between the previous values and the COSY derived values in both the measured and predicted cases is similar. Technical difficulties prevented accurate tracking through the 11th beam position monitor. Therefore most tracking studies were only performed up through RC9. These technical issues prevented a full examination of the tracking predictions, but the data that was taken can be used as a tool to diagnose misalignments [25]. Since the steering solution is off-center in most of the ring elements, all nonlinear effects are present due to feed-down. Hence, a good agreement between measured and predicted steering solutions is indicative of an accurate modeling of the full nonlinear dynamics.

The COSY predicted settings compared with the UMER calculated settings, as well as another set of UMER settings based on a LOCO-type response matrix steering algorithm [9], were used to provide a common basis for comparison of the steering solutions; see Fig. 15. The

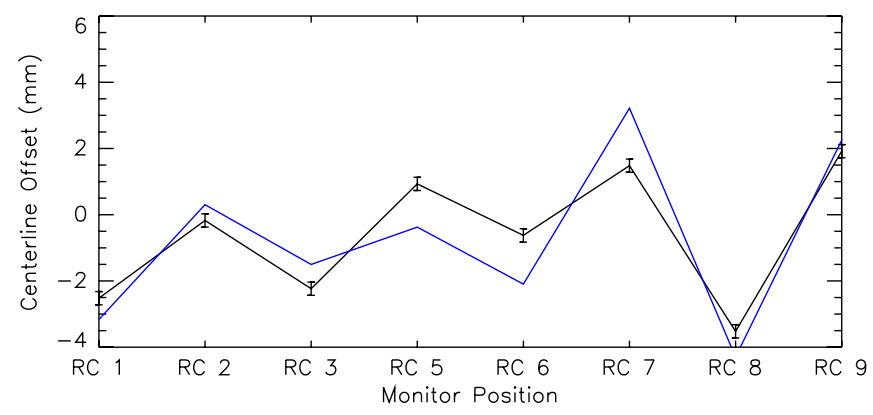

FIG. 15. (Color) Steering solution using the default values for the UMER ring; a comparison of measured (black) to predicted values (blue).

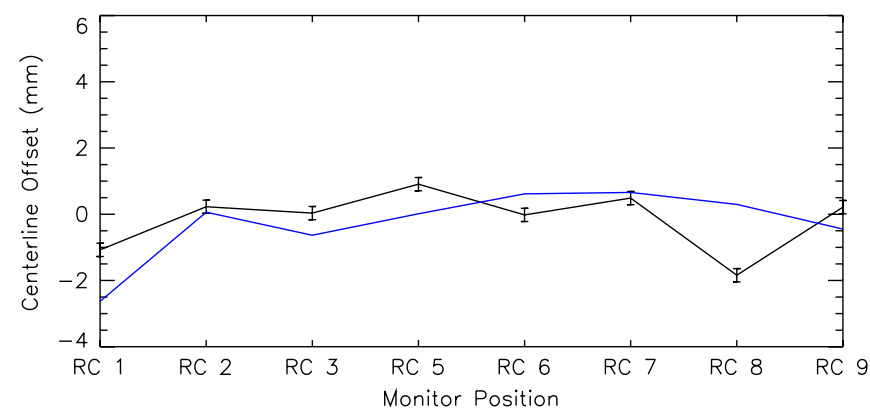

FIG. 16. (Color) The blue line is the predicted value for the new steering settings, while the black line is the measured trajectory.

original steering solution in COSY assumed that the beam would be injected straight along the centerline, which is not necessarily the case, so a new steering solution was devised based on the UMER stock matching settings. As can be seen in Fig. 16, there was significant improvement.

\section{SUMMARY AND CONCLUSIONS}

This study undertook an analysis of the single particle nonlinear effects in the University of Maryland electron ring, both through simulation and follow-up experiments. The abilities of the software package COSY INFINITY allow for a multitude of fast, accurate simulations. The University of Maryland electron ring has a number of unique qualities including effects from the earth's magnetic field, which were modeled throughout the ring using measured data. In simulations, we studied beam steering, matching, betatron tunes, chromaticities, resonance strengths, momentum compaction, and dispersion. The results of these studies show that UMER has rich nonlinear dynamics even in the absence of space charge. We showed that steering in the nontrivial geometry of the injection and recirculation section can be done accurately. We also showed that the closed orbit distortions could be minimized to less than $1 \mathrm{~mm}$ horizontally and $\sim 5 \mathrm{~mm}$ vertically. We identified some of the best operating points where the beam survival is maximized over many turns.

In summary, the simulations and analyses of the simulations showed that a wide variety of behavior could be observed simply by varying the currents to the two large quadrupoles within the injection line. Some settings were clearly superior to others, showing both better beam survival, better resilience to placement offsets, and greater robustness with respect to magnet current offsets. Furthermore, COSY's ability to calculate quantities such as relative resonance strengths might be able to explain why operating points act differently under many turns, including why the $7 \mathrm{~mA}$ beam seems to be the most stable of the possible beams being used.

Some simulation analyses were implemented and were analyzed on the actual machine. Benchmarking involved observing the beam trajectory as it moved through the various beam position monitors. When compared with 
simulated data, the beam agrees well with measurements made in the ring through the first half of the machine. It is noteworthy that, not only do the trajectories within the first half of the ring match closely, it is actually possible to use the COSY model to improve the steering of the ring in a nontrivial manner. A more complete benchmarking, using the full ring, is in progress.

Of course, the primary purpose of UMER is to study space-charge dominated beams, so our comprehensive nonlinear model of the ring will serve as an excellent starting point for space-charge related simulations. An analysis of the interplay between the single particle nonlinear dynamics and the effects of space charge will be very interesting. Furthermore, the relative importance of intensity-dependent effects are the subject of ongoing studies.

\section{ACKNOWLEDGMENTS}

This work was supported by the Department of Energy under Contract No. DE-FG02-08ER41532 with Northern Illinois University. UMER's funding comes from the Department of Energy offices of High Energy Physics and Fusion Sciences. The help and cooperation of UMER was greatly appreciated, both with equipment familiarization and experiment design. Furthermore we would like to especially thank R. Kishek, D. Sutter, S. Bernal, W. Chao, B. Beaudoin, and K. Fiuza.

\section{APPENDIX A: EARTH'S MAGNETIC FIELD KICK}

This is the derivation for the kick strength used to model the earth's magnetic field. In the notation of Sec. II A, $\vec{g}_{1}$ and its corresponding solution $\mathcal{M}$ is already known and implemented in COSY for all magnetic elements represented in the UMER ring. The function $\vec{g}_{2}$ and its corresponding solution $\mathcal{K}$ is due to the earth's magnetic field. The standard, canonical COSY coordinates are used, $\vec{z}=$ $(x, a, y, b)$, where $a=p_{x} / p_{0}$ and $b=p_{y} / p_{0}$, the coordinates $x$ and $y$ are the horizontal and vertical positions, $\vec{p}=$ $\left(p_{x}, p_{y}, p_{s}\right)$ is the momentum of the particle, and $p_{0}$ is the momentum of the reference particle. These coordinates are chosen to be implemented using Strang splitting, since these are the coordinates that COSY uses to express the transfer maps. For the earth's field inside each element, we assume constant values equal to the value at the center of the element, $\vec{B}=\left(B_{x}, B_{y}, B_{z}\right)=$ constant. The relevant differential equations for the kick are [13]

$$
\begin{gathered}
x^{\prime}=0, \\
a^{\prime}=\left[b \frac{B_{z}}{\chi_{m 0}}-\frac{B_{y}}{\chi_{m 0}}\right](1+h x), \\
y^{\prime}=0,
\end{gathered}
$$

$$
b^{\prime}=\left[\frac{B_{x}}{\chi_{m 0}}-a \frac{B_{z}}{\chi_{m 0}}\right](1+h x),
$$

where $h=\frac{1}{r}, r$ being the radius of curvature, and $\chi_{m 0}$ is the magnetic rigidity of the reference particle. Assuming initial conditions $\left(x_{i}, a_{i}, y_{i}, b_{i}\right)$, the solution is

$$
\begin{gathered}
x(s)=x_{i}, \\
a(s)=\left(b_{i}-\frac{B_{y}}{B_{z}}\right) \sin \left(\frac{B_{z}}{\chi_{m 0}}\left(1+h x_{i}\right) s\right) \\
+\left(a_{i}-\frac{B_{x}}{B_{z}}\right) \cos \left(\frac{B_{z}}{\chi_{m 0}}\left(1+h x_{i}\right) s\right)+\frac{B_{x}}{B_{z}}, \\
y(s)=y_{i}, \\
b(s)=\left(\frac{B_{x}}{B_{z}}-a_{i}\right) \sin \left(\frac{B_{z}}{\chi_{m 0}}\left(1+h x_{i}\right) s\right) \\
+\left(b_{i}-\frac{B_{y}}{B_{z}}\right) \cos \left(\frac{B_{z}}{\chi_{m 0}}\left(1+h x_{i}\right) s\right)+\frac{B_{y}}{B_{z}} .
\end{gathered}
$$

For sections that do not bend $h=0$. Finally, the kick $\mathcal{K}$ is given by (A5)-(A8) evaluated at $s=\ell$.

\section{APPENDIX B: IMAGE CHARGE KICK}

In order to properly account for the effects of image forces on the beam, it becomes important to determine the magnitude of the force in order to apply a kick. We assume a perfectly conducting straight cylindrical beam pipe, and a beam which has cylindrical symmetry, with a constant longitudinal charge density that is long enough for end effects to be neglected; see Fig. 17. This is a good approximation for UMER. Using a cylindrical coordinate system the differential equation is of the form

$$
\ddot{\xi}-\frac{q \lambda}{2 \pi \epsilon_{0} m} \frac{\xi}{R^{2}-\xi^{2}}=0,
$$

where $\xi$ is the offset from the center of the beam pipe, $R$ is the radius of said beam pipe, $q$ is the charge of an electron, $\lambda<0$ is the charge density along the beam pulse, $m$ is the mass of the electron, and $\epsilon_{0}$ is the electric permittivity. This models the forces on the centroid of the beam.

Since the differential equation is of the form $\xi^{\prime \prime}+$ $G^{\prime}(\xi)=0$, it has the conservation law,

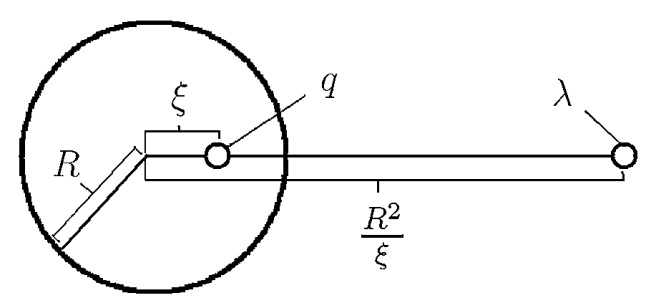

FIG. 17. Diagram of the coordinate system in use for deriving the effects of image charge on the motion of the beam. 


$$
\frac{1}{2} \xi^{\prime 2}+G(\xi)=E .
$$

We assume $\xi^{\prime}=0$ at $\xi=0$, since the image charge in a cylinder only affects off-center beams. This leads to

$$
\xi^{\prime}= \pm \sqrt{-\frac{q \lambda}{2 \pi \epsilon_{0} m} \ln \left(\frac{R^{2}-\xi^{2}}{R^{2}}\right)}
$$

where $\xi$ is the magnitude of the displacement and equal to $\sqrt{x^{2}+y^{2}}$. Using $a, b$ to represent the angles while assuming that the particles are nonrelativistic, we get their kick strength equal to

$$
\begin{aligned}
& \Delta a=\frac{x}{v_{0} \sqrt{x^{2}+y^{2}}} \sqrt{-\frac{q \lambda}{2 \pi \epsilon_{0} m} \ln \left(\frac{R^{2}-\left(x^{2}+y^{2}\right)}{R^{2}}\right)}, \\
& \Delta b=\frac{y}{v_{0} \sqrt{x^{2}+y^{2}}} \sqrt{-\frac{q \lambda}{2 \pi \epsilon_{0} m} \ln \left(\frac{R^{2}-\left(x^{2}+y^{2}\right)}{R^{2}}\right)} .
\end{aligned}
$$

Now we derive the equation of motion for an individual (test) electron in the beam so that the image force can be applied to an arbitrary set of initial conditions. So we define the variable $Y$ as the line between the particle in question and the image charge; see Fig. 18. Once again we get that

$$
\ddot{\Upsilon}-\frac{q \lambda}{2 \pi \epsilon_{0} m} \frac{1}{\Upsilon}=0
$$

This is solved in a similar manner to the centroid problem:

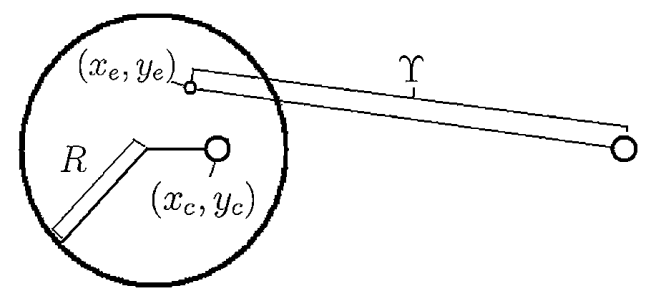

FIG. 18. Diagram of the coordinate system in use for deriving the effects of image charge on a test electron in the beam.

$$
\dot{Y}=\sqrt{2 C+\frac{q \lambda}{\pi \epsilon_{0} m} \ln (Y)}
$$

Now we define the coordinates of the image charge and the particle. The position of the image charge is based on the location of the beam centroid with coordinates $\left(x_{c}, y_{c}\right)$, and the location of the test electron will be $\left(x_{e}, y_{e}\right)$. This leads to

$$
\begin{aligned}
& \Upsilon_{x}=\frac{R^{2} x_{c}}{\xi^{2}}-x_{e} \\
& \Upsilon_{y}=\frac{R^{2} y_{c}}{\xi^{2}}-y_{e}
\end{aligned}
$$

where in the notation just introduced $\xi=\sqrt{x_{c}^{2}+y_{c}^{2}}$. This entails

$$
\sqrt{Y_{x}^{2}+Y_{y}^{2}}=\sqrt{\left(\frac{R^{2}}{\xi}\right)^{2}-2 \frac{R^{2}}{\xi}\left(\frac{x_{e} x_{c}+y_{e} y_{c}}{\xi}\right)+x_{e}^{2}+y_{e}^{2}}
$$

Inserting this into (B7) we obtain

$$
\dot{Y}=\sqrt{C+\frac{q \lambda}{\pi \epsilon_{0} m} \ln \left[\sqrt{\left(\frac{R^{2}}{\xi}\right)^{2}-2 \frac{R^{2}}{\xi}\left(\frac{x_{e} x_{c}+y_{e} y_{c}}{\xi}\right)+x_{e}^{2}+y_{e}^{2}}\right]} .
$$

To determine the value of $C$ we require that if the test electron coincides with the centroid of the beam, $x_{e}=x_{c}, y_{e}=y_{c}$, then $\dot{Y}=\dot{\xi}$. The solution for $C$ is reinserted in the equation, where we apply the unit vectors to get the direction of the kick, and we scale it to the particle optical coordinates $a$ and $b$. Finally, we obtain

$$
\begin{gathered}
x(s)=x_{i}, \\
a(s)=a_{i}+\frac{\frac{R^{2}}{\xi} x_{c}-\xi x_{i}}{\sqrt{R^{4}-2 R^{2}\left(x_{i} x_{c}+y_{i} y_{c}\right)+\left(x_{i}^{2}+y_{i}^{2}\right) \xi^{2}}} \sqrt{\frac{q \lambda}{2 \pi \epsilon_{0} m} \ln \left(\frac{R^{6}-2 R^{4}\left(x_{i} x_{c}+y_{i} y_{c}\right)+\left(x_{i}^{2}+y_{i}^{2}\right) R^{2} \xi^{2}}{\left(R^{2}-\xi^{2}\right)^{3}}\right) \frac{1}{v_{0}},} \\
y(s)=y_{i},
\end{gathered}
$$




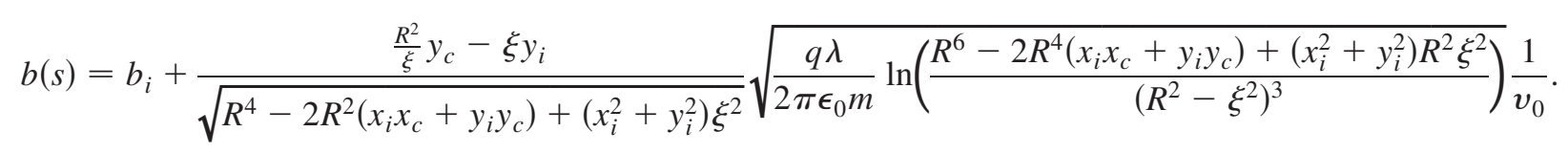

The kick $\mathcal{K}$ is given by evaluating at $s=\ell$.

From a programming perspective, it is possible when fitting, for $\xi$ to become greater than $R$. For this reason the software was written so that if $\xi$ was greater than $R$, the procedure would return a zero kick, and the objective function for the kick would be increased by a set amount.

[1] R. A. Kishek et al., Nucl. Instrum. Methods Phys. Res., Sect. A 561, 266 (2006).

[2] R. A. Kishek et al., Int. J. Mod. Phys. A 22, 3838 (2007).

[3] I. Haber et al., Nucl. Instrum. Methods Phys. Res., Sect. A 606, 64 (2009).

[4] L. G. Vorobiev, X. Wu, and R. York, in Proceedings of the 18th Particle Accelerator Conference, New York, 1999 (IEEE, New York, 1999), pp. 3116-3118.

[5] S. Bernal, D. Sutter, M. Cornacchia, B. Beaudoin, I. Haber, R. Kishek, M. Reiser, C. Wu, and P. O'Shea, in Proceedings of the 13th workshop on Advanced Accelerator Concepts (AAC) (AIP Press, New York, 2009), pp. 738-743.

[6] S. Bernal et al., in Proceedings of the 23rd Particle Accelerator Conference, Vancouver, Canada, 2009 (IEEE, Piscataway, NJ, 2009).

[7] C. F. Papadopoulos, R. Fiorito, P. O'Shea, A. Shkvarunets, M. C. W. Gai, and J. G. Power, in Proceedings of the 23rd Particle Accelerator Conference, Vancouver, Canada, 2009 (Ref. [6]).

[8] D. Sutter, S. Bernal, C. Wu, M. Cornacchia, B. Beaudoin, K. Fiuza, I. Haber, R. Kishek, M. Reiser, and P. O'Shea, in Proceedings of the 23rd Particle Accelerator Conference, Vancouver, Canada, 2009 (Ref. [6]).

[9] C. Wu, E. H. Abed, B. Beaudoin, S. Bernal, K. Fiuza, I. Haber, R. A. Kishek, P. G. O'Shea, M. Reiser, and D.
Sutter, in Proceedings of the 23rd Particle Accelerator Conference, Vancouver, Canada, 2009 (Ref. [6]).

[10] R. A. Kishek, S. Bernal, C. L. Bohn, D. Grote, I. Haber, H. Li, P. G. O'Shea, M. Reiser, and M. Walter, Phys. Plasmas 10, 2016 (2003).

[11] H. Li, S. Bernal, T. Godlove, R. Kishek, P. O'Shea, and M. Reiser, in Proceedings of the 19th Particle Accelerator Conference, Chicago, Illinois, 2001 (IEEE, Piscataway, NJ, 2001), p. 1802.

[12] K. Makino and M. Berz, Nucl. Instrum. Methods Phys. Res., Sect. A 558, 346 (2006).

[13] M. Berz, Advances in Imaging and Electron Physics (Academic Press, London, 1999), Vol. 108.

[14] E. Hairer, C. Lubich, and G. Wanner, Geometric Numerical Integration: Structure Preserving Algorithms for Ordinary Differential Equations (Springer-Verlag, Berlin, 2002).

[15] A. J. Dragt (unpublished).

[16] B. Erdelyi, Ph.D. thesis, Michigan State University Center for Dynamical Systems and Mathematical Physics, 2001.

[17] S. Blanes, F. Casas, J. Oteo, and J. Ros, Phys. Rep. 470, 151 (2009).

[18] S. Blanes, F. Casas, and A. Murua, Boletin de la Sociedad Española de Mathemática Aplicada (2008), pp. 89-145.

[19] A. Bátkai, P. Csomós, B. Farkas, and G. Nickel, arXiv: $1002.0164 \mathrm{v} 2$.

[20] S. Bernal, Technical Report No. 060306-SB, UMER (2006).

[21] S. Bernal, Technical Report No. 060220r-SB, UMER (2006).

[22] M. V. et al., Fusion Eng. Des. 32-33, 283 (1996).

[23] M. Venturini, Ph.D. thesis, University of Maryland Physics Department, 1998.

[24] D. Sutter (private communication).

[25] E. Nissen, B. Erdelyi, S. Bernal, and D. Sutter, in Proceedings of the 23rd Particle Accelerator Conference, Vancouver, Canada, 2009 (Ref. [6]). 